\title{
The red cell osmometer
}

\section{A useful inaccuracy in measurement of mean corpuscular volume in hyperosmolar states}

\author{
SC ALLEN,* IC BALFOUR, $\dagger$ AND CC WISE $\ddagger$ \\ From the *Department of Anaesthetics, University Hospital of Wales, Heath Park, Cardiff CF4 $4 X N$ and the \\ $\dagger$ Department of Haematology and $\ddagger$ Intensive Therapy Unit, Cardiff Royal Infirmary, Cardiff CF2 1SZ, UK
}

SUMmaRY The Coulter S Counter gives a value for mean corpuscular volume which does not necessarily reflect red cell size in vivo. This phenomenon may be used in early diagnosis and hence prevention of the 'hyperosmolar syndrome'.

This in vitro study arose from observations made in the intensive therapy unit. It had been noted that a raised mean corpuscular volume (MCV), as measured by the Coulter Counter Model $\mathrm{S},{ }^{*}$ could coexist with hypernatraemia in certain states of hyperosmolality.

The postulated underlying mechanism was that the rate of movement of osmotically active particles, particularly d-glucose, into the intracellular compartment exceeded their removal by normal intracellular metabolism. The result of this state would be water shift to produce an expanded intracellular space, a depleted extracellular space, and a rise in plasma sodium, reflecting the overall rise in osmolality. The observed rise in MCV would therefore reflect this pattern, and the MCV would function as an indicator of intracellular osmolality.

The Coulter $\mathbf{S}$ Counter is a widely used instrument in haematology laboratories. The MCV is measured by determination of the mean pulse height generated during the period of electronic cell counting calibrated by reference to known blood specimens. The 'Coulter Principle' of one-by-one counting and sizing of particles is now well established. The excellent degree of precision of the measurement has also been established by Sharp and Ballard. ${ }^{1}$

Before measurement the sample is diluted 50000 times with Isoton II* solution. Isoton II is a buffered saline diluent intended for counting and

*Coulter, Coulter Counter Model S, and Isoton II are registered trade marks of Coulter Electronics Limited.

Received for publication 22 October 1979 sizing blood cells. It contains preservatives and stabilisers used to give long storage life and stability of MCV after dilution of blood specimens for up to 30 minutes. Its osmolality, as measured by freezing point depression, is approximately $345 \mathrm{mOsm} / \mathrm{kg}$. The preservatives and stabilisers contribute to this apparently high result by this measurement procedure but do not appear to affect the measured MCV. Blood cells in Isoton II can be shown to be similar in size to blood cells in normal saline at the time of dilution, although exact comparisons are impossible because blood cells swell rapidly and progressively when suspended in normal saline. ${ }^{2-6}$ It is therefore believed that Isoton II has an effective osmolality of $300 \mathrm{mOsm} /$ $\mathrm{kg}$. For normal use in a Coulter Counter, a satisfactory diluent does not have to be 'isotonic' provided it gives stable results after dilution, since the MCV result is calibrated under those same conditions from known fresh blood specimens. ${ }^{7}$ It is this dilution which produces the inaccuracy of MCV measurement in conditions where the red cell being processed is originally hypertonic. Since the extracellular environment is changed by such a large dilution in the Coulter, the red cell becomes isotonic with the diluent.

If the cell is originally hypertonic due to removal of water by an osmotic agent added to the extracellular environment, water transfer will occur on dilution until the red cell has the same size as an untreated cell in the same diluent. The MCV as measured by the Coulter Counter will be the same in both cases. Similarly, if the hypertonic cell contains osmotically active particles which can diffuse freely out of the cell in the 30-second period of dilution, the MCV as measured by the Coulter 
Counter will again be the same as that of a normotonic sample.

However, if the red cell contains molecules which cannot freely diffuse back across the cell membrane, water will be extracted from the diluent to produce a larger cell isotonic with the extracellular diluent. Therefore, an apparent increase in MCV as measured by the Coulter Counter is produced.

The same principles apply whatever the osmolality of the diluent. We have taken samples of blood in vitro and made them hyperosmolar by the addition of known hypertonic solutions. We have then compared MCV measurements made directly by a 'manual' method and by the Coulter S Counter.

\section{Method}

Thirty millilitre samples of blood were taken from healthy volunteers and anticoagulated with 300 units of heparin solution; $3 \mathrm{ml}$ aliquots of blood were added to tubes each containing $0.3 \mathrm{ml}$ of known hypertonic solutions. The concentration of each additive had been calculated to increase plasma osmolality by $100 \mathrm{mOsm}$. The hypertonic solutions used were $20 \%$ mannitol, $6.6 \%$ urea, $20 \%$ d-glucose, and $20 \%$ l-glucose. Two control samples were used. One control contained no additive, the other $0.3 \mathrm{ml} 0.9 \% \mathrm{NaCl}$. In later experiments, where plasma sodium concentration was measured, an additional control sample diluted with $0.3 \mathrm{ml} 5 \%$ mannitol was used. The latter acted as an isotonic control (measured osmolality $283 \mathrm{mOsm} / \mathrm{kg}$ ) which contained neither dextrose nor sodium.

All samples were mixed for 1 hour at room temperature. Each was then processed through the

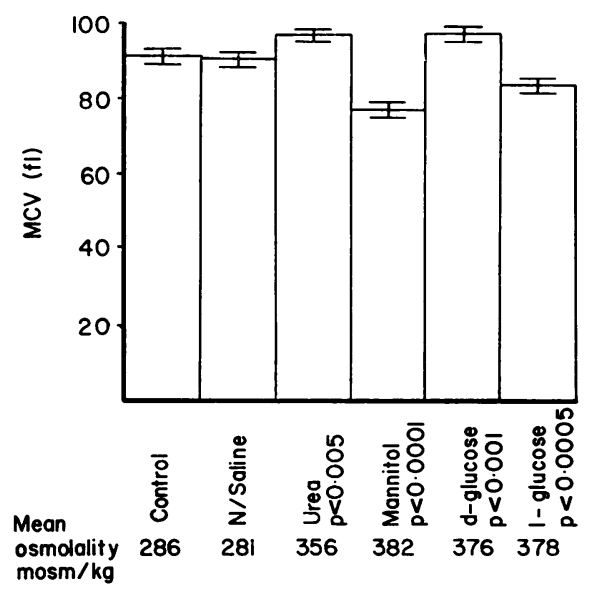

Fig. 1 Manual MCV: mean values \pm 2 standard errors. $\mathrm{P}$ values compared with control.

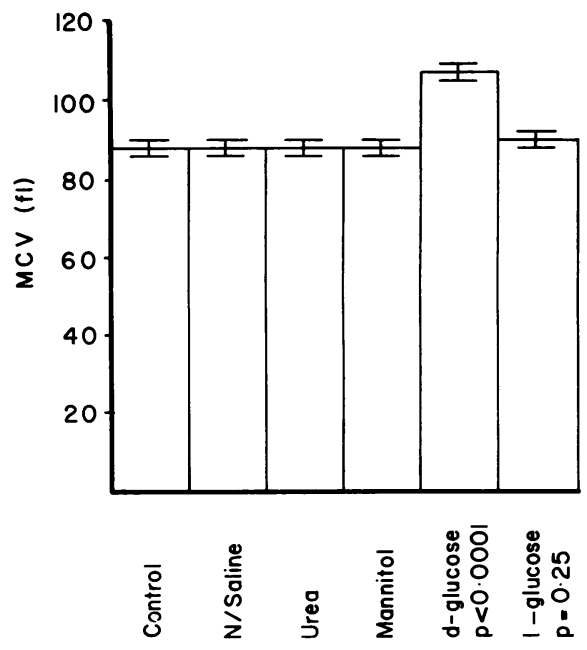

Fig. 2 Coulter MCV: mean values \pm 2 standard errors. P values compared with control.

Coulter S Counter, and microcapillary tube samples were taken for packed cell volume (PCV) estimation using a high-speed microcentrifuge. Calculation of 'manual' MCV was made using the red cell count from the Coulter Counter. Recordings of Coulter MCV and 'manual' MCV were made. Plasma sodium concentrations were measured using a standard flame photometer technique. Plasma osmolality was measured in a number of samples using the method of depression of the freezing point (Advanced Instruments Inc Model 3L).

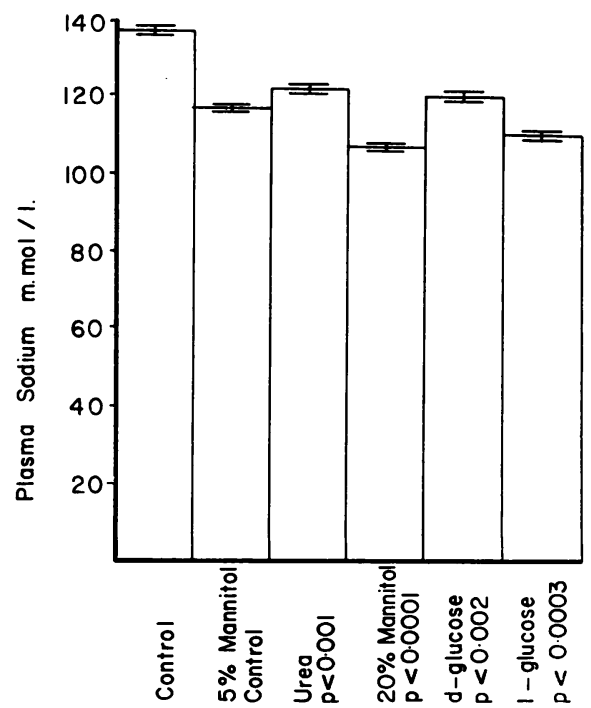

Fig. 3 Plasma sodium levels: mean values \pm 2 standard errors. P values compared with $5 \%$ mannitol control. 
Comparison of observed and predicted values of plasma sodium levels, using mean values from pooled data

\begin{tabular}{lllllll}
\hline & Control & Mannitol control & $20 \%$ Mannitol & Urea & d-Glucose & l-Glucose \\
\hline $\begin{array}{c}\text { Observed value } \\
\text { (mmol/l) }\end{array}$ & 137 & 117 & 122 & 107 & 120 & 110 \\
$\begin{array}{c}\text { Predicted value } \\
\text { (mmol/l) }\end{array}$ & - & 116 & 120 & 107 & 121.5 & 111.5 \\
\hline
\end{tabular}

\section{Results}

These are summarised in Figs 1 to 3 . The mean values are plotted plus or minus 2 standard errors. Statistical analysis of results was performed using the unpaired $t$ test.

The results provide an interesting and very simple demonstration of osmosis across the red cell membranes.

'MANUAL' METHOD

There was no significant difference between the undiluted control sample and that diluted with normal saline. It was essential to our experiment to produce consistent results of 'manual' MCV since we are assuming that the 'manual' method is the accurate representation of red cell size in vivo.

When d-glucose is added, the red cell becomes larger as glucose and water enter the cell. Mannitol produces a fall in MCV as intracellular water is removed. L-glucose is not metabolically active and produces an effect similar to, but smaller than, that of mannitol. A $6.6 \%$ urea solution produced a rise in 'manual' MCV, suggesting that the red cell actively concentrates urea.

\section{COULTER METHOD}

There was no significant difference between the samples with any agent other than d-glucose. The previously noted changes of red cell size had been reversed by the transfer of water into the cell (mannitol, l-glucose) or of small molecules out of the cell (urea).

In the case of d-glucose, however, the previously noted rise in MCV was exaggerated as further water shifted into the intracellular compartment.

\section{SODIUM ESTIMATIONS}

The shifts in plasma water are also demonstrated by plasma sodium levels (Fig. 3). The control value, using $5 \%$ mannitol, is low due to the dilution of the sample. Changes from this control value with other agents are small but significant. Plasma sodium is raised when water shift into the cells occurs with d-glucose and urea, and lowered with mannitol and l-glucose as water is removed from the cell.
The observed values for plasma sodium are close to those predicted by calculation from the 'manual' PCV (Table).

\section{Discussion}

The Coulter S Counter produces seven indices on its printout. The MCV is probably given little attention by many clinicians despite its precision. Our work shows that the method has discrepancies which appear to have been unrecognised until now, and that recognition of the nature of these gives valuable clinical information.

The differences between 'manual' MCV and control values and the changes of plasma sodium are consistent with the postulated shifts of water between the intracellular and extracellular compartments. The inverse relationship between changes of 'manual' MCV and plasma sodium measurements indicates the direction in which water has moved. Thus, mannitol and l-glucose cause a shift of water out of the cell while urea and d-glucose increase intracellular water.

The Coulter MCV results for each group demonstrate the change in red cell size produced by dilution of the measured sample during the automatic counting process. All the groups except d-glucose showed no significant change from the control value. The shifts of water and additive molecules causing the changes in 'manual' MCV must therefore be freely reversible when the extracellular environment of the red cells is replaced by Isoton II. This is not true for d-glucose, where the molecule or its metabolites are 'held' inside the cell. The hypertonic cell causes water shift into the cell when diluted, and a consequent further rise in MCV as measured by the Coulter Counter.

Surprisingly, a $6.6 \%$ urea solution produced a rise in 'manual' MCV. Urea was included in our study to demonstrate the action of a molecule which we supposed passed freely across cell membranes. Were this so, then there should be no change in 'manual' MCV compared with the control. The rise in 'manual' MCV we cannot explain, except that it must be produced by a water shift into the cell as a result of a higher concentration of urea intracellularly than in the plasma. The Coulter MCV for 
urea is unchanged from the control, showing that the urea molecules rapidly pass back across the cell membrane into the Coulter diluent.

It is interesting to note that the addition of 1-glucose produces a smaller change in 'manual' MCV than that of mannitol, despite similar changes in plasma osmolality. There is likewise a smaller change in plasma sodium. The Coulter MCV is higher (although this last change is not significantly different from the control). This might suggest that a small quantity of l-glucose does cross the red cell membrane at these concentrations, or that some d-glucose was present as a contaminant.

In conventional haematological disorders, the MCV is raised in pathological situations leading to macrocytic red cells as, for example, in megaloblastic anaemia and liver disease. Our results show that if the cell contains an excess of small molecules the 'manual' MCV will be increased. If these molecules cannot diffuse back across the red cell membrane, the MCV will be even further increased by dilution with Isoton II before the Coulter estimation. Changes of osmolality in the patient due to changes in urea, body water, or administration of mannitol will not affect the Coulter MCV, although our experiment shows that the Coulter MCV does not give correct measurement of red cell size in vivo. Where hyperosmolality is secondary to glucose intake, the actual MCV would be expected to be high and the Coulter MCV to give an even higher value.

Red cells have a life of 120 days. Unless transfused, critically ill patients have largely the same cells throughout their stay in the intensive care unit. Serial MCV measurements will then indicate the cause of hyperosmolality if this is secondary to excessive carbohydrate administration. We suggest that serial measurement of Coulter MCV in parallel with plasma sodium is of value in the elucidation of the cause of hyperosmolality.

We thank the Haematology Department, Cardiff Royal Infirmary, and the Biochemistry Department, University Hospital of Wales, for technical assistance. We are also very grateful for the interest and advice given by Dr PJF Griffiths, Department of Chemistry, University of Wales Institute of Science and Technology, and by Mr RW Lines, of Coulter Electronics Ltd.

\section{References}

${ }^{1}$ Sharp AA, Ballard BCD. An evaluation of the Coulter S Counter. J Clin Pathol 1970;23:327-35.

2 Blades AM, Flavell HCG. Observations of the use of the Coulter Model ' $D$ ' Electronic Cell Counter in clinical haematology. J Clin Pathol 1963;16:158-63.

${ }^{3}$ Brecher G, Schneiderman M, Williams GZ. Evaluation of electronic red blood cell counter. Am J Clin Pathol 1956;26:1439-49.

4 Brecher G, Jakobek EF, Schneiderman MA, Williams GZ, Schmidt PJ. Size distribution of erythrocytes. Ann NY Acad Sci 1962;99:242-61.

${ }^{5}$ Frederick GL. Effect of different diluents on electronic determination of erythrocytic volume frequency distribution curves of farm animals. Can J Physiol Pharmacol 1964;42:680-3.

${ }^{6}$ Nevius DB. Osmotic error in electronic determinations of red cell volume. Am J Clin Pathol 1963;39:38-41.

${ }^{7}$ Personal communication. RW Lines, Coulter Electronics Ltd.

Requests for reprints to: Dr SC Allen, Department of Anaesthetics, University Hospital of Wales, Heath Park, Cardiff CF4 4XN, UK. 